\section{Modern Microscopy On the Light Side}

\author{
Skip Palenik, Senior Research Microscopist \\ McCrone Associates, Inc.
}

Developments in microscopy in the last twenty years or so I have been dramatic and impressive. Not only can individual atoms be seen with relatively inexpensive instruments, but is even possible to I manipulate them, fulfilling the prophecies made by Richard Feyman I in his "Room at the Bottom" address given in 1959*. Why, one might I reasonably ask, when it is possible to observe, manipulate, and identify single atoms, would someone want to waste their time with an instrument so "old fashioned" as a light microscope?

The fact is that most problems submitted for microscopical I study do not occur at the atomic level. From preliminary inspection through sample preparation and final identification, light microscopes i are indispensable tools for scientists throughout industry in general, as I well as such specialized fields as art conservation and forensic sciI ence. It is not infrequently true, however, that microscope users (not all are microscopists) from one discipline can not talk to those from I another. This is unfortunate since the techniques utilized in one disI cipline may be quite useful to someone in another field, if they just I knew about them.

I

Many of the microscopical methods currently in common use were originally developed by microscopists working in disciplines I other than those with which they are commonly associated today. I Polarized light microscopy is perhaps the best known example of this. - Originally developed by Sorby and a long list of followers as a means for studying the mineral composition and fabric of rocks in thin secI tion, it has evolved well beyond the study of minerals (to which it is I still applied) to fields far removed from anything Sorby could have i imagined. Thus it is routinely used in the identification and i characterization of chemical crystals of all sorts, synthetic polymers, I air pollutants and both natural and synthetic fibers. A similar case I can be made for other microscopical methods. Incident light I microscopy was originally developed as a technique for studying the microstructure of polished metal surfaces (also by Sorby, as it turns I out), but has been extended to such remote fields as the examination I of ores and cement. Probably the single greatest use of incident light I microscopy today is in the semiconductor industry, where it is I ! essential in the inspection of silicon wafers and integrated circuits. Still another example is fluorescence microscopy which was originally developed almost as a curiosity. Crude ultraviolet sources directed to I the stage of a microscope have given way to highly efficient systems I for directing the exciting rays to the specimen by means of dichromatic (not dichroic, as incorrectly stated in some manufacturers , literature) mirrors, which just as efficiently transmit the longer I fluorescent light emitted from the specimen to the observer. Because I of their extreme sensitivity, histochemical reactions with fluroescently labeled reagents have become indispensable in modern medical research and diagnosis. The same approach holds promise for the I industrial microscopist wishing to demonstrate the location of a cation I or functional group in a thin section of polymer, although it is rarely used in this way.

In future columns we will try to point out, by example, some I of the applications of modern light microscopy in a contemporary re- I search setting. Plans include articles on fibers, fluorescence and multilaminate polymer films which will describe both techniques and interpretation in a concise, yet brief, format.

* For a recent summary of this classic talk see "The Man Who Dared to Think Small," Science 254: 29 November 1991, p. 1300-1.

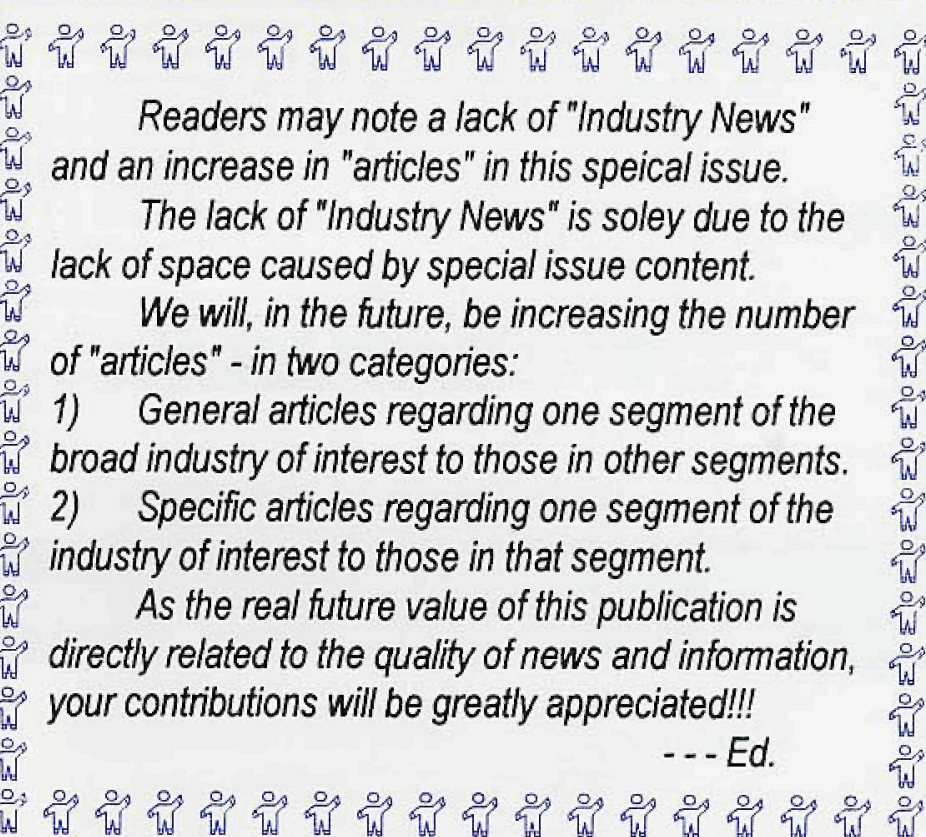

SOFTWARE FOR

STM \& AFM DATA ANALYSIS

\section{KEVEX}

world leader in microanalysis

25 th anniversary celebration

EMSA booths $704-720$

come join us

FISONS

Instruments

A NEW DATA REDUCTION METHOD WHICH PRODUCES

PARAMETERS WITH CLEAR PHYSICAL INTERPRETATIONS FOR PRACTICAL APPLICATIONS IN DESIGN, ANALYSIS AND MANUFACTURE OF SUB-MICRON TOPOGRAPHIES

based on: SURFACE AREA - SCALE RELATIONS using: FRACTAL ANALYSIS BY PATCHWORK TRIANGLES

FOR MORE INFORMATION AND SAMPLE ANALYSES CONTACT: BURLINGTON COMPUTER SYSTEMS developed by: C.A. Brown, P.E., Ph.D and Associates PATENT PENDING
PO BOX 249

WATERBURY CENTER, VT 05677 802 244-1554 